\title{
Research about Monitoring Systems of Special Roads Based on 4G
}

\author{
Jian $\mathrm{Luo}^{1, \mathrm{a}}$,Xi Zhu ${ }^{2, \mathrm{~b}}$ and Xiaoqing $\mathrm{Hou}^{3, \mathrm{c}}$ \\ ${ }^{1}$ School of Chang'an University, Xi'an 710064,China, \\ ${ }^{2}$ School of Chang'an University, Xi'an 710064,China, \\ ${ }^{3}$ School of Chang'an University, Xi'an 710064,China. \\ aluojian@chd.edu.cn, ${ }^{\mathrm{b}}$ houxiaoqing0525@126.com, ${ }^{\mathrm{c}} 925990173 @ q q . c o m$.
}

Keywords: Special roads, Video monitoring,4G.

\begin{abstract}
Monitoring systems of special roads based on 4G start point video monitoring systems designing of special roads. Combined with special roads of several major features, it is concluded that the ideal way of information transmission is $4 \mathrm{G}$ communication technology, and through the monitoring method research of traffic flow, combined with the structure of the monitoring system and 4G video transmission technology, the ideal special roads traffic flow monitoring and information transmission system can be designed. For 4G communication technology application in highway monitoring system it has important foresee and practical guiding significance, and for the special roads of traffic flow monitoring it has a very important role.
\end{abstract}

\section{Preface}

With the development of national economy, transportation is playing an increasingly important role in economic development. Transportation safety is getting more and more attentions in the special roads. It's necessary to set up monitoring systems to monitor the traffic flow data and ensure the traffic safety. About special road, it usually refers to road which is beyond normal variations in designing limits and under the influence of the special climate condition or geographical conditions including landform and physiognomy, such as extra-long highway tunnel, extra-long bridge, long downhill, geological disasters (earthquakes, landslides) road, city T-junction, etc. Because of the relatively poor linear, big traffic flow, bad driving behavior of drivers, vehicle performance and other factors in these roads, it's very easy to cause traffic accidents. It's necessary to set up monitoring systems to monitor the traffic flow data and ensure the traffic safety. 4G technology not only supports images, music, video and other media forms of wireless transmission, but also has the advantages of high transmission rate, good adaptability, low cost, etc. Using 4G wireless transmission technology can solve the problem of repaved communication pipelines. It is a kind of new type and high quality way of information transmission. Therefore, using the research about monitoring systems of special roads on $4 \mathrm{G}$ can fully make advanced modern network technology and communication technology apply to traffic management. So it can better achieve the goal of traffic information real-time and two-way transmission to reduce the occurrence of the traffic incidents.

\section{The monitoring system of special roads}

\subsection{The summarize of monitoring system}

Highway monitoring system is one of the most important parts of the highway electromechanical systems, which can quickly, accurately and completely collect the information of road. By analyzing the monitoring data of traffic information and using information release facilities to convey information to road users, it can make the road users understand the road conditions in a timely manner. Highway monitoring system can ensure the smooth traffic and road safety, which is the important means to realize highway operation and management ${ }^{[1]}$.

Traditional highway monitoring system is mainly composed of information collection subsystem, video monitoring subsystem, information subsystem, traffic control subsystem and computer network system, etc. As shown in Fig.1. 


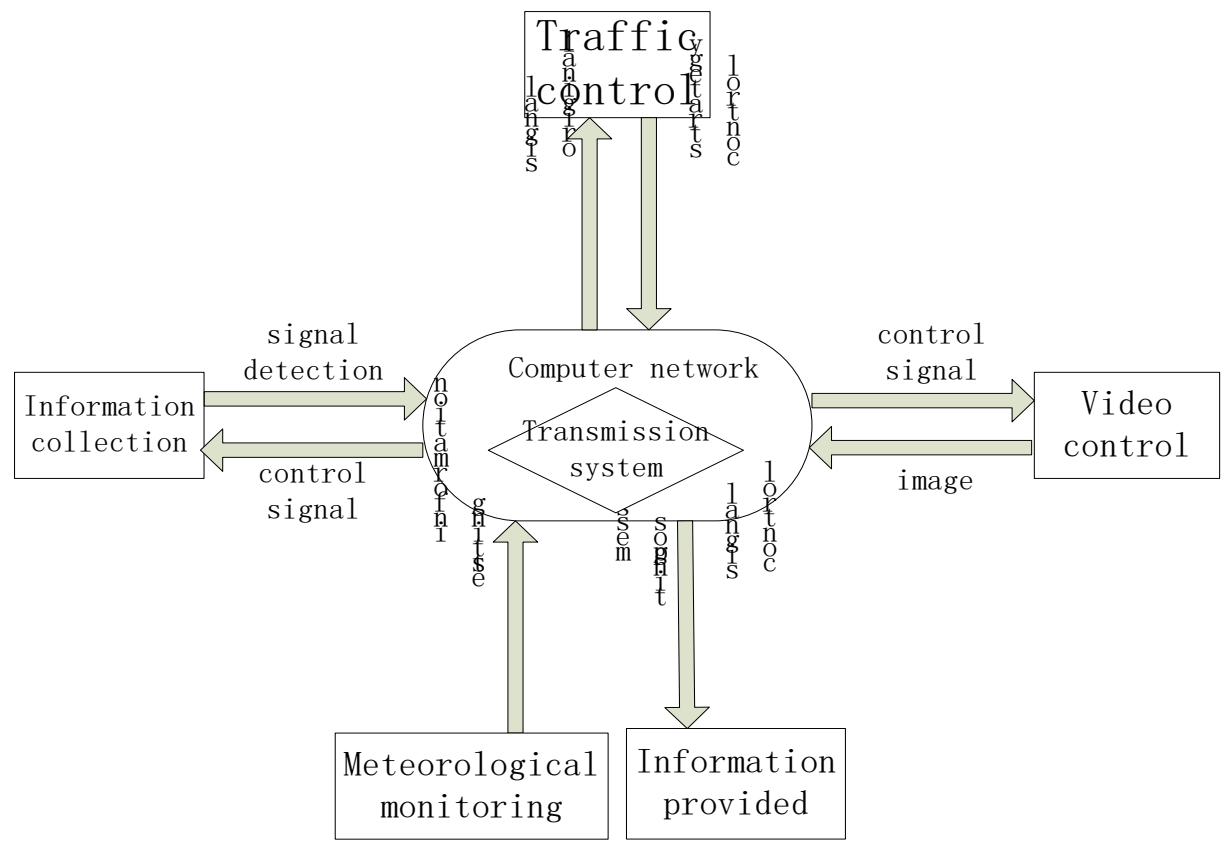

Fig.1 Monitoring and control system structure diagram

Usually we put a camera on certain sections of the highway, and make it monitored, using the system to monitor the extent of the traffic. For special roads, it's not easy to establish the perfect monitoring system. So you can understand the traffic conditions through the establishment of relatively simple and easy video monitoring system. According to the existing highway monitoring system structure, and special roads of their own road conditions, the monitoring and control system can be simplified, whose basic structure is divided into front-end equipment, signal transmission parts and monitoring terminal ${ }^{[2]}$. Front-end used to capture images or video of the monitored area, generally composed of the camera and lens, decoder, dust cover and so on. Transmission part of the camera output video and audio signals to a central computer room or other monitoring points. Terminal is used for displaying, recording, video processing and control signal outputting, generally including a monitor records, all kinds of equipment and control equipment, etc.

\subsection{The front-end collection subsystem}

As the first function modules of the monitoring system, the front-end acquisition subsystem is the front of the whole system. The quality of image acquisition will directly affect the effect of the whole system, and the speed of acquisition is the key to the whole system design. In addition, the effectiveness and stability of the image data compression also affect the quality of the monitoring image to the terminal display and storage. Therefore it plays a vital role in effective operation of the monitoring system. What is shown in Fig.2 is the structure diagram for the front-end acquisition subsystem.

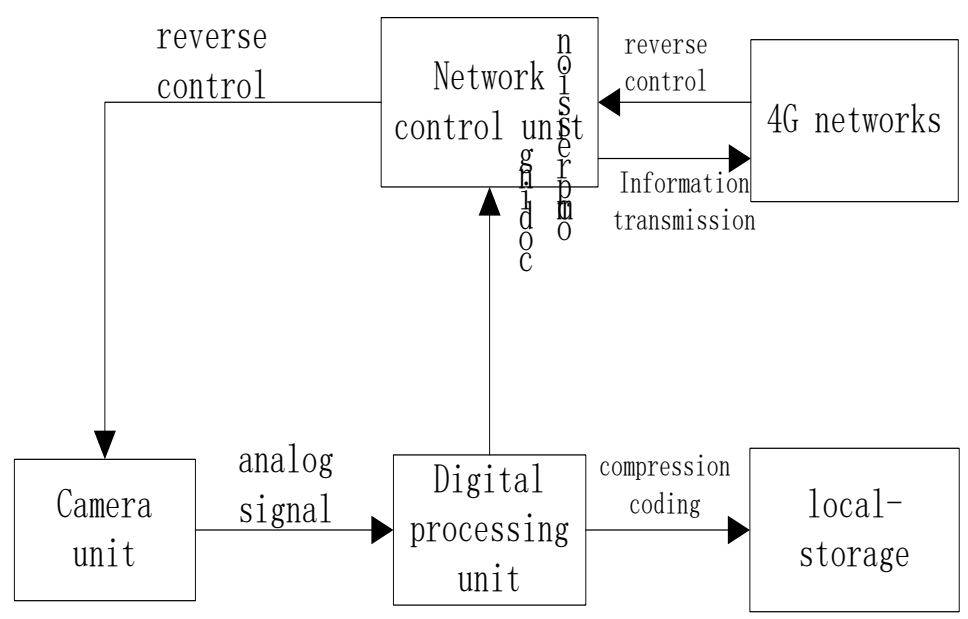

Fig.2 Front-end structure diagram 
Camera unit will absorb things light signal into electrical signal, usually using a CCD camera. And CCD is short for charge-coupled device. After video compression coding, it will access the 4G network for network transmission. We can choose $4 \mathrm{G}$ router to achieve the goal of front-end data connection with 4G network transmission ${ }^{[3]}$. After front-end network video server network outputting the image stream with cable, it will connect to $4 \mathrm{G}$ router. Then connected by a router with 4G base stations, data code can be circulated to $4 \mathrm{G}$ networks for network transmission. At the same time, the router can receive signals from terminal monitoring center of the front control, and transfer to the network video server to control and operate the lens, etc.

2.3 The terminal supervisory control subsystem

The terminal monitoring control system functional block diagram is shown in Fig.3 as follows.

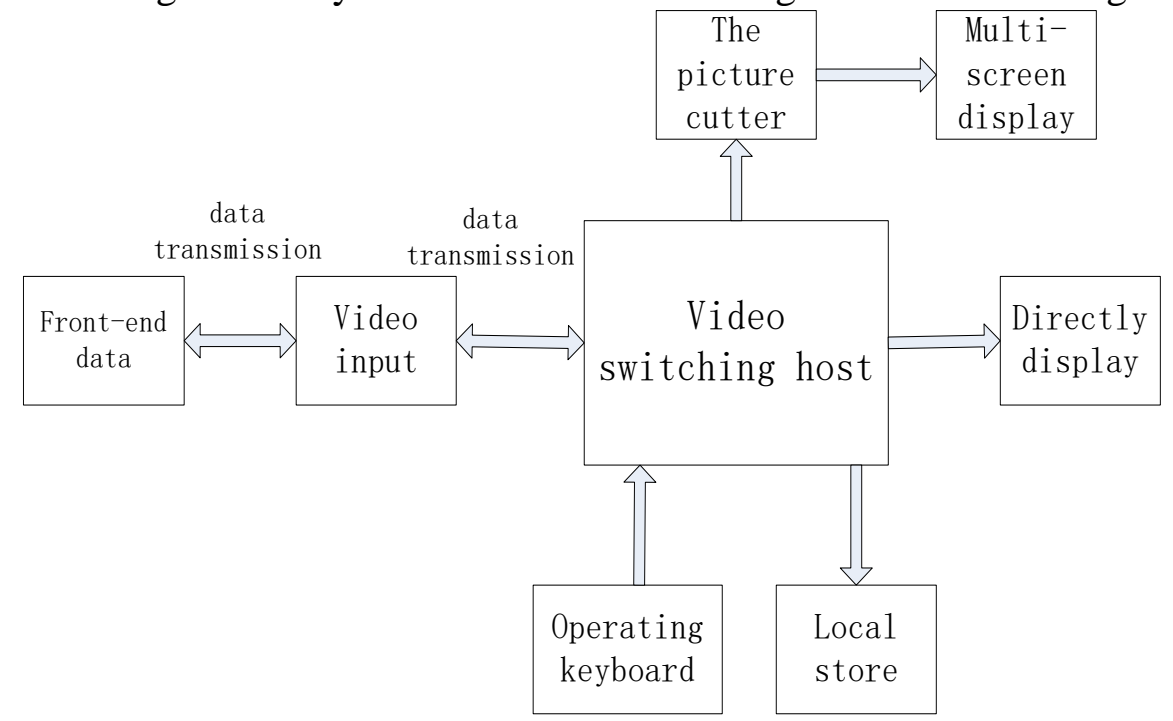

Fig.3 Terminal functional block diagram

After front-end data through the 4G network transmission to the monitoring center, the video input interfaces. After processing by the video switch mainframe, on the one hand, data will write to the local hard drive. On the other hand, after the processing of the picture cutter, multiple monitoring information can be realized on the big screen display. At the same time for single important video information, it can be directly call display.

\section{4G network transmission}

\subsection{The analysis of $4 \mathrm{G}$ communication network and demand}

Communication network is the bridge of data acquisition and data processing ${ }^{[4]}$, which can transfer the original data information to the traffic monitoring center by cable or wireless network transmission. After the monitoring center processing the raw data, it will get the information for further analysis. Through that it can judge the road traffic congestion problems. When traffic congestion is going to occur, it will release traffic information, and adopt bypass measures in a timely manner to dredge traffic and prevent traffic from congestion.

Currently cable transmission used in the intelligent transportation system mainly adopts the standard RS - 232 or optical fiber communication, etc. The traditional cable transmission is very inconvenient in the special roads, which are far away from the monitoring center. So we hope which is wireless transmission scheme at a low cost and high reliability to replace the traditional way of cable transmission. With low cost, low power consumption, high speed, flexible networking, etc,4G can facilitate the wireless connection between devices. The communication construction is convenient and the power supply can use battery or solar panels, so it's the ideal choice to realize wireless data acquisition system.

\subsection{The transmission structure of monitoring network based on 4G}

The front-end collection system firstly collects the image, then code and compress it. After that, the image becomes the convenient-transferred data stream through the internet, which at the same time conforms to according protocol. The front-end can access to 4G internet through setting the 
terminal of $4 \mathrm{G}$ router. Data stream transfer to router, then pass through some according network configuration, like the gateway and addressing. Next, pass through the wireless channel; finally arrive to the wireless access point, which we call the 4Gbase.

Communication network transmission diagram as shown in Fig.4.

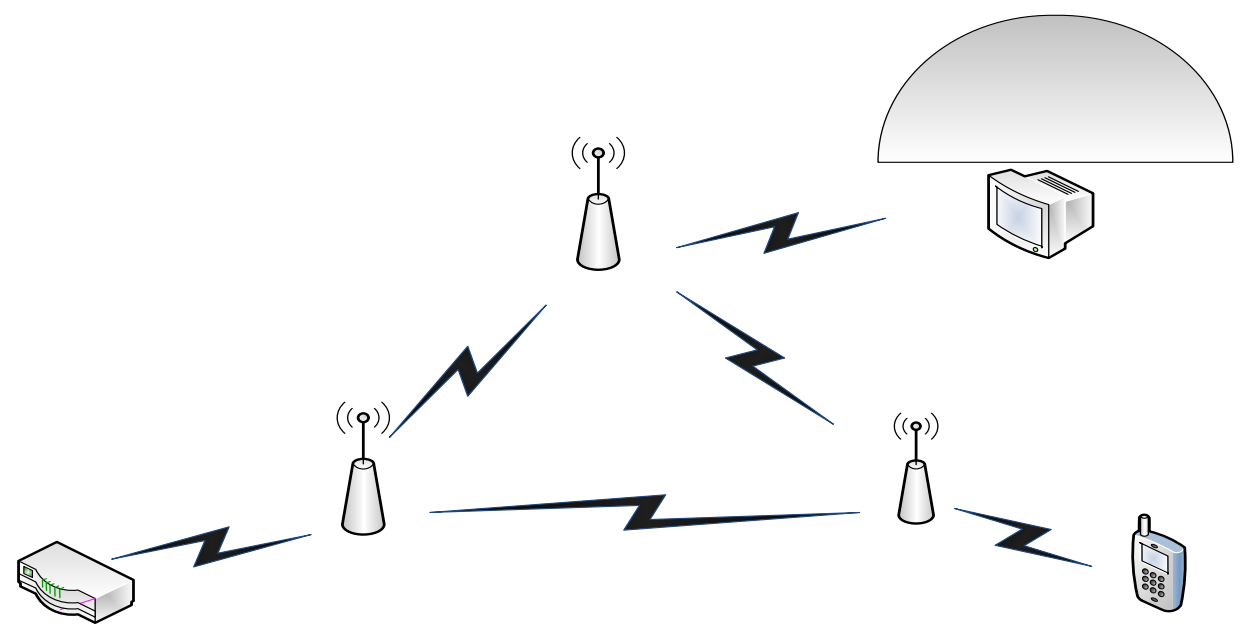

Fig.4 Network transmission diagram

As is shown, the signal is sent to the $4 G$ network access point via a front-end router, after transmission in the network, according to the need. On the one hand it can be transferred to your phone / PAD and other 4G terminal equipments, which can achieve simple display monitoring. On the other hand, it's also the main that data transfers to the monitoring center and the monitoring image in the center of the monitor display related functions such as storage, playback and control. AP is 4G network access points, which establish communication with the system two-way connection with front-end 4G router. On the one hand, AP receives the data from the acquisition front-end code stream, on the other hand also transfer control information from the monitoring center to assist controlling front-end collection equipment. As a wired to wireless transmission device, front-end routers need to configure the router and set the network parameters, and at the same time can also build the monitoring center network ${ }^{[5]}$.

\section{Conclusions}

From the introduction, the article firstly analysis the present situation of monitoring system and points out the problem that the special road monitoring system urgently needs to be researched and break through. Then, through the study of the demand analysis of transmission system, we find the ideal transmission mode. Finally, we design up monitoring and control system structure and network scheme of the traffic flow based on the technology.

About the core of this article 4G video monitoring system, mainly consists of the front-end acquisition subsystem, network transmission system and terminal monitoring subsystem ${ }^{[6]}$. In addition, it also supports reverse monitoring control, which makes the whole monitoring system more intelligent.

With the development of $4 \mathrm{G}$ wireless network technology, coupled with the use of some new technology, its implementation and application will provide a good transportation support for the development of social economy. It can be true for some special sections area to solve the problem of traffic monitoring laid, and to play an important role of huge boost to the development of road monitoring. As technology becomes more mature, 4G networks using cost reduces and mature technology tends to be more stable, 4G network monitoring system will become a popular choice of wireless real-time monitoring. 


\section{References}

[1] Zhongjie Zhao,Shiyan Xu.Highway Monitoring System.Peking: China Communications Press,2014,p.2-5.

[2] Rong Ye,Kun Zhao.The Design of the Special Highway Roads Monitoring Facilities. China Transportation Information,Vol.9 (2013) No.9,p.112.

[3] Xiangmo Zhao,Yinli Ji. Highway Monitoring System of Theory and Application. Peking: Electronic Industry Press,2013,p.2-5.

[4] Xiuling Wei,Zhengjin Wang. Traffic Flow Detection Transfer Research Based on 3G. Digital Technology and Application, Vol.7(2012) No.7,p.28-29.

[5] Video Image Transmission Based on 4G: www.Google.com.

[6] Yonghong Ddeng. The Summarize of 4G Communication Technology. Digital Communication World,Vol.2(2005) No.5,p.65. 